\title{
The effect of vitamin D Treatment on COVID 19- patients, an inverted propensity score weighting (IPSW), and inverted probability of treatment weighting (IPTW) analyzed study
}

Jamal Wadi Al Ramahi ${ }^{1}$, Nour Hasan², Ammal Matar ${ }^{2}$, Ma'en Maher Al-Ali ${ }^{3}$, Lara Abdulhadi², Dania Abu Kaf ${ }^{3}$, Waseem Saadeh ${ }^{4}$, Nour Hamdan ${ }^{4}$, Hassan AbuKhalaf ${ }^{4}$, Mohamed Gharaibeh ${ }^{5}$, Hanadi Hamadallah ${ }^{6}$, Ala'abader ${ }^{7}$, Mohammad Atout ${ }^{4}$, Sae'ed Moh. Mar' ${ }^{4}$, Tamer Alhamed ${ }^{4}$

\section{Abstract}

Background: Vitamin D3 $\left(1,25(\mathrm{OH})_{2}\right.$ cholecalciferol) as a treatment for COVID 19 patients is being disputed, and a clear clinical benefit is not being confirmed.

Methods: A retrospective evaluation for COVID-19 patients who were treated with various cumulative doses of vitamin D. Data was extracted from the COVID-19 database, it included patients admitted to three hospitals in Amman, Jordan. Characteristics of patients were tabulated and compared for all-cohort, and propensity score index (PSI) adjustment. The comparison was based on two vitamin D strata ( $\leq 149,000$ i.u. and $>150,000$ i.u.). Logistic regression analysis was utilized to predict recovery, the need for oxygen, and all-cause mortality for all-cohort, IPSW, and IPTW patients, based on vitamin D cumulative doses during their hospital stay.

Results: 1131 all-cohort and 768 PSI-adjusted patients were recruited. Except for antibiotics and antivirals, all other characteristics were balanced $(P=N S)$. There were 1017 patients on vitamin $D, 847$ received cumulative $\leq 149,000$ i.u., and 170 patients received cumulative dose
1 Department of Medicine, School of Medicine, The University of Jordan. Amman, Jordan

2 Al Khalidi Hospital, Clinical Pharmacy, Amman, Jordan

3 Department of Clinical Medical Sciences, Faculty of Medicine, Yarmouk University, Irbid, Jordan.

4 Department of Medicine. The Specialty Hospital. Amman, Jordan.

5 Department of Anesthesia, The Specialty Hospital. Amman, Jordan.

6 Jordan hospital, Department of Pharmacy. Amman, Jordan.

7 The Specialty hospital. Department of pharmacy. Amman, Jordan.

Contact information:

Jamal Wadi AI Ramahi, MD, FIDSA.

झjamalwadimd@yahoo.com 
$\geq 150,000$ i.u. (Range $1000-385000$ ). It was demonstrated that the escalation of the cumulative doses of vitamin $D$ did not contribute to the assessed outcomes; all-cohort patients (OR $=1.000,95 \%$ C.I. 1.000 to 1.000$)$, IPSW (OR $=1.000,95 \%$ C.I. 1.000 to 1.000$)$, and the IPTW $(O R=1.000,95 \%$ C.I. 1.000 to 1.000$)$.

Conclusion: In our patients' cohorts, we could not demonstrate a beneficial effect for vitamin D therapy in COVID-19 patients in recovery, the need for home oxygen, and all-cause mortality, by hospital discharge.

\section{Keywords}

Vitamin D; COVID-19;

COVID-19 Recovery; Covid-19

Mortality; COVID-19 the need

for home oxygen therapy.

\section{Introduction}

COVID-19 hit the world populations causing morbidities and mortalities, cure and/or mitigation are being sought. Several interventions are adopted in the treatment of COVID-19 without strong evidence, like an effective antiviral therapy [1-3], zinc and ascorbic acid [4], and convalescent plasma [5, 6]. Vitamin D3 $\left(1,25(\mathrm{OH})_{2}\right.$ cholecalciferol) is being used in COVID-19 patients for its known immunomodulating effects, and its role in signaling during adaptive and innate immune responses in viral, and bacterial infections [7]. Previous studies associated the initial high serum levels, and vitamin D supplementations with reduced incidence of seasonal influenza or improvement in the duration and severity [8-10]

A large population-based observational study included 39,190 COVID-19 patients with low serum vitamin D levels $(<20 \mathrm{ng} / \mathrm{ml})$ had had higher positivity for SARS-CoV-2 (12.5\%) compared with 27,870 patients with higher levels where they had lower positivity (8.1\%), even 12,321 patients with values $\geq 55 \mathrm{ng} / \mathrm{mL}$ had positivity rates of $5.9 \%$, in a multivariate analysis, the association rates between positivity rates and higher circulation Vitamin $D$ was demonstrated (AOR 0.984 per ng/ml increment, $P<0.001)$ [11]. However, the use of vitamin $D$ in COVID-19 patients have been linked to uncertainty, evidence supporting its use appeared as it was derived from a small RCT study detecting a reduction in COVID-19 severity in ICU patients, similar evidence came from other observational studies [12-14]

In the current study, we aim to illustrate the treatment effects of using vitamin D in COVID-19 patients. Our patients were prescribed different and varying doses during their hospital stay, to avoid confusion about regimens the total cumulative doses for vitamin $D$ were evaluated to predict causal treatment effects.

\section{Materials and Methods}

\section{Study Settings}

Data for COVID-19 patients was collected from three participating hospitals (The Specialty, Jordan, and Al Khalidi) with a total bed capacity of around 700 , special units for the management of patients with COVID-19 were allocated with an approximate capacity of 155-floor beds and 47 ICU beds. The study was a retrospective cross-sectional over 22 weeks (28 November 2020 to 6 May 2021), data was uploaded into a cloud excel sheet (Microsoft Corporation). Records were included as patients presented for admissions in the participating hospi- 
tals. The study was approved by each of the internal review boards of the three hospitals, no consent was needed.

\section{Treatment protocols}

There is a current updated COVID-19 management protocol published by the Jordan Ministry of Health $(\mathrm{MOH})$. In the three hospitals, the treating physicians partially relied on the $\mathrm{MOH}$ protocol and literature updates, the treatment protocol for vitamin D was heterogeneous (supplementary material); different doses, and may vary over the treatment period in the same patient. Vitamin D tablets (used as a three-day 50,000 I.U. regimen before March 15, then a seven-day 50,000 I.U., also a 2000 I.U. and 5000 I.U., daily were prescribed. Other agents administered; steroids (dexamethasone or solumedrol), anticoagulants (Enoxaparin sodium, Apixaban, Rivaroxaban, and Fondaparinux), antivirals (Favipiravir, Remdesivir), acetylsalicylic acid (ASA), colchicine, Zn tablets, and Vitamin C (supplementary material).

\section{Classification of radiological findings}

Chest radiography scoring system: the degree of lungs involvement was based on radiologists' classification for the degree of lungs involvement. Normal chest x-ray and/or normal CT chest with no infiltrate were considered as no involvement (score 1), a lobar infiltrate with $25 \%$ involvement (score 2), scattered ground glass appearance involving lungs with $>25$ $50 \%$ involvement (score 3), diffuse patchy infiltrate $>50-75 \%$ involvement was considered as (score $4)$, and multilobe infiltrate was considered as $>75 \%$ involvement (score 5) [15].

\section{Statistical analysis}

Characteristics for the all-cohort and PS-adjusted patients were described, propensity-score match tolerance (caliper) was 0.1, without replacement, normal distribution for predictors was tested by skewness, histogram, P-P blot, and Q-Q blot, all fitted a normal curves distribution, skewness for all were $<1.0$ and $>-1.0$, multicollinearity was evaluated by linear regression (tolerance was above 0.251 , mostly $>0.8$ and VIF was mostly less than 1.3 except two with 3.554 and 3.992), continuous predictors were evaluated by Levine's test and were homoscedastic. Predicted probability was derived from continuous and binary predictors thru binary logistic regression analysis, some continuous predictors were Log10-transformed to normalize the distribution before they were incorporated in the model (supplementary material). Predictors entered were: Age, gender, the sum of recorded symptoms, comorbidities, Log_BMI, Tobacco, Log_ferritin level, Log_D-Dimer, Log_LDH, imaging scores, steroids, antivirals, antibacterials, Antifungal, IL6-inhibitors, documented temperature, blood oxygen saturation, oxygen delivery method, and peripheral white blood cells $[16,17]$. Vitamin $D$ treatment was analyzed by Chi-square test $\left(\chi^{2}\right)$ astwo categories $(<149,000,>$ $150,000)$ with post hoc analysis by Bonferroni adjusted $p$-value to assure balanced variables. IPSW patients were estimated for the outcome effects by Logistic regression analysis, it was tabulated for the all-cohort, PSM, and SIPTW patients. SPSS version 25 with Python Essentials and Fuzzy extension command blog-ins was used in the analysis, significance was considered for values $<0.05$.

\section{Results}

The characteristics of the 1220 patients were reviewed, eighty-nine cases had missing data and were excluded. Analysis was for 1131 as the allcohort, and 768 PSM and 768 PS-adjusted patients (Table 1). The differences in characteristics between two vitamin $\mathrm{D}$ dose categories among the all-cohort were balanced with the PSI (Table 1), few characteristics had significant $(P<0.05)$ but borderline imbalance with PS-adjustment. Age was balanced in the initial cohort except for the age group (Lowest thru 65 years), more patient proportions were in the higher vitamin D cumulative dose group $(\leq 149,000$ i.u. 
Table 1. The characteristics of COVID-19 patients according to Vitamin D treatment allocations.

\begin{tabular}{|c|c|c|c|c|c|c|c|}
\hline \multirow{4}{*}{\multicolumn{2}{|c|}{ Characteristic }} & \multirow{2}{*}{\multicolumn{3}{|c|}{$\begin{array}{c}\text { All cohort, Vitamin D } \\
\qquad N=1017\end{array}$}} & \multicolumn{3}{|c|}{ Propensity score-adjustedpatients } \\
\hline & & & & & \multicolumn{3}{|c|}{$N=768$} \\
\hline & & \multirow{2}{*}{$\begin{array}{c}\leq 149,000 \text { i.u. } \\
n=847\end{array}$} & \multirow{2}{*}{$\begin{array}{c}\geq 150,000 i . u \\
n=170\end{array}$} & \multirow{2}{*}{$\mathbf{P}$ * } & $\leq 149,000$ i.u. & $\geq 150,000$ i.u. & \multirow{2}{*}{$P$ * } \\
\hline & & & & & $n=639$ & $n=129$ & \\
\hline \multirow{3}{*}{ Age (years) } & Lowest thru 65 & $481(56.9)$ & $112(65.9)$ & \multirow{3}{*}{0.072} & 372 & 82 & \multirow{3}{*}{0.429} \\
\hline & 66 thru 75 & 194 & 34 & & 139 & 27 & \\
\hline & 76 thru highest & 171 & 24 & & 128 & 20 & \\
\hline \multirow{2}{*}{ Gender } & Male & 544 & 405 & \multirow{2}{*}{0.542} & 413 & 82 & \multirow{2}{*}{0.817} \\
\hline & female & 303 & 65 & & 226 & 47 & \\
\hline \multicolumn{2}{|l|}{ Antivirals } & $537(67.3)$ & $73(43.7)$ & $<0.05$ & $443(69.3)$ & $58(45)$ & $<0.05$ \\
\hline \multicolumn{2}{|l|}{ Antibiotics } & $656(77.4)$ & $81(47.6)$ & $<0.05$ & $504(78.9)$ & $62(48.1)$ & $<0.05$ \\
\hline \multicolumn{2}{|l|}{ Antifungal } & 68 & 20 & 0.197 & 57 & 18 & 0.195 \\
\hline \multicolumn{2}{|c|}{ Colchicine } & 205 & 31 & 0.217 & 166 & 25 & 0.114 \\
\hline \multicolumn{2}{|c|}{ Presenting symptoms (Sum) } & 847 & 170 & NS & 639 & 129 & NS \\
\hline \multicolumn{2}{|c|}{ Documented fever } & 833 & 168 & 0.606 & 669 & 131 & 0.651 \\
\hline \multirow{6}{*}{ Comorbidities } & Two or more & 439 & 80 & \multirow{6}{*}{0.857} & 349 & 63 & \multirow{6}{*}{0.851} \\
\hline & Diabetes mellitus & 49 & 12 & & 35 & 11 & \\
\hline & Chronic lung disease & 11 & 1 & & 11 & 1 & \\
\hline & Heart disease & 10 & 2 & & 9 & 2 & \\
\hline & Hypertension & 70 & 12 & & 51 & 9 & \\
\hline & Malignancy & 7 & 0 & & 4 & 0 & \\
\hline \multirow{4}{*}{ BMI } & Lowest thru 25 & 176 & 26 & \multirow{4}{*}{0.142} & 146 & 21 & \multirow{4}{*}{0.123} \\
\hline & $26-30$ & 295 & 71 & & 240 & 60 & \\
\hline & 31 thru highest & 230 & 48 & & 190 & 43 & \\
\hline & Tobacco use & 99 & 12 & & 80 & 10 & \\
\hline & $>94$ & 174 & 34 & & 117 & 21 & \\
\hline Blood & $90-94$ & 286 & 70 & & 216 & 54 & \\
\hline $\begin{array}{l}\text { oxygen } \\
\text { saturation }\end{array}$ & $85-89$ & 184 & 35 & 0.179 & 149 & 29 & 0.380 \\
\hline (\%) & $80-84$ & 102 & 19 & & 85 & 16 & \\
\hline & $<80$ & 88 & 9 & & 72 & 9 & \\
\hline & RA & 121 & 15 & & 88 & 9 & \\
\hline & Simple mask & 108 & 26 & & 72 & 21 & \\
\hline & High flow & 25 & 11 & & 20 & 8 & \\
\hline Oxygen & Noninvasive ventilation & 34 & 4 & 0318 & 29 & 3 & 0516 \\
\hline $\begin{array}{l}\text { delivery } \\
\text { method }\end{array}$ & Combined & 36 & 7 & 0.318 & 30 & 7 & 0.516 \\
\hline & IMV\% & 31 & 2 & & 24 & 2 & \\
\hline & Non-rebreather mask & 168 & 30 & & 142 & 25 & \\
\hline & Nasal Prongs & 309 & 72 & & $245(36.6)$ & $56(42.7)$ & \\
\hline
\end{tabular}




\begin{tabular}{|c|c|c|c|c|c|c|c|}
\hline \multirow{4}{*}{\multicolumn{2}{|c|}{ Characteristic }} & \multirow{2}{*}{\multicolumn{3}{|c|}{$\begin{array}{c}\text { All cohort, Vitamin D } \\
\qquad N=1017\end{array}$}} & \multicolumn{3}{|c|}{ Propensity score-adjustedpatients } \\
\hline & & & & & \multicolumn{3}{|c|}{$N=768$} \\
\hline & & $\leq$ 149,000i.u. & $\geq 150,000$ i.u. & \multirow{2}{*}{$\mathbf{P}$ * } & $\leq 149,000$ i.u. & $\geq 150,000$ i.u. & \multirow{2}{*}{$P$ * } \\
\hline & & $n=847$ & $n=170$ & & $n=639$ & $n=129$ & \\
\hline \multirow{5}{*}{$\begin{array}{l}\text { Imaging } \\
\text { score } \\
\text { (X-ray and } \\
\text { CT) }\end{array}$} & Normal & 41 & 7 & \multirow{5}{*}{0.253} & 24 & 3 & \multirow{5}{*}{0.166} \\
\hline & $25 \%$ Involvement & 60 & 10 & & 49 & 9 & \\
\hline & $\begin{array}{l}>25-50 \% \\
\text { Involvement }\end{array}$ & 302 & 69 & & 243 & 57 & \\
\hline & $\begin{array}{l}>50-75 \% \\
\text { Involvement }\end{array}$ & 231 & 55 & & 165 & 40 & \\
\hline & $>75 \%$ Involvement & 206 & 28 & & $156(24.4)$ & $19(14.7)$ & \\
\hline \multirow{2}{*}{$\begin{array}{l}\text { Procalcitonin } \\
\text { level ( } \mathrm{ng} / \mathrm{mL} \text { ) }\end{array}$} & $\leq 0.5$ & $286(33.8)$ & 91(53.5) & \multirow{2}{*}{$\begin{array}{c}<0.05 \\
\text { NS }\end{array}$} & 245 & 75 & \multirow{2}{*}{0.866} \\
\hline & $>0.5$ & 101 & 26 & & 82 & 24 & \\
\hline \multirow{3}{*}{$\begin{array}{l}\text { D-Dimer } \\
(\mathrm{ng} / \mathrm{mL})\end{array}$} & $\leq 0.5$ & 287 & 66 & \multirow{3}{*}{0.249} & 212 & 51 & \multirow{3}{*}{0.323} \\
\hline & $>0.5-2$ & 293 & 63 & & 241 & 48 & \\
\hline & $>2$ & 209 & 33 & & 181 & 30 & \\
\hline \multirow{3}{*}{$\begin{array}{l}\text { Ferritin } \\
\mathrm{ng} / \mathrm{ml}\end{array}$} & $<260$ & 133 & 20 & \multirow{3}{*}{0.451} & $10.3(16.1)$ & $12(9.3)$ & \multirow{3}{*}{0.131} \\
\hline & $260-1000$ & 374 & 74 & & 294 & 62 & \\
\hline & $>1000$ & 297 & 67 & & 242 & 55 & \\
\hline
\end{tabular}

Percent in (brackets) are proportions ofsub-variables when statistical significance was demonstrated.*: 2-sided Significance (P-value) was tested by $\chi 2$, and Bonferroni method.\% Invasive mechanical ventilation. NS: not significant.

$=56.9 \%$ versus $\geq 150,000$ i.u. $=65.9 \%, p<0.05)$, however, all other categories were balanced in the PS adjustment. Gender remained well-balanced in the initial cohort $(P=0.542)$ and the PS-adjusted patients $(P=0.817)$. Antivirals and antibiotics were imbalanced in both all-cohort and the PS-adjusted patients $(P<0.05)$. Documented fever, comorbidities, BMI, tobacco use, and blood oxygen saturation were well balanced for both cohorts $(P=N S)$. Oxygen delivery method was balanced for the allcohort patients and PS-adjustment ( $P=N S$ ) except for the nasal prongs, it remained imbalanced $(P<$ $0.05)$ where more patients were in the vitamin $D$ category $>150,000$ i.u. Admission radiological imaging showed well balance in the all-cohort and PSadjusted patients except it were imbalanced for the $>75 \%$ involvement. PCT levels were well balanced in both cohorts $(P=N S)$, but imbalance was in the subcategory $(\leq 0.5 \mathrm{ng} / \mathrm{ml})$. D-Dimer levels were well balanced in both cohorts $(P=N S)$. Serum ferritin was imbalanced for the subcategory $(<260 \mathrm{ng} / \mathrm{ml})$ in the PS-adjusted patient, both cohorts were well balanced $(P=N S)$.

There were 1017 patients on vitamin D, 847 received cumulative $\leq 149,000$ i.u., and 170 patients received cumulative dose $\geq 150,000$ i.u. (Dose range $1000-385000$ ), and the majority (823) was in $\leq$ 50,000 cumulative i.u.

\section{Outcomes analyses}

In the all-cohort analysis, recovery was in 401 patients, vitamin $D$ supplement increasing cumulative dose did not significantly contribute to recovery ( $\mathrm{B}$ $=0.000, \mathrm{~S} . \mathrm{E}=0.000$, Wald $=4.076, \mathrm{P}=0.043$, $\mathrm{OR}=1.000,95 \%$ C.I. 1.000 to 1.000 ), also, no significance was noted for IPSM $(B=0.000, S . E=$ 0.000, Wald $=187.905, P=0.000, O R=1.000$, 95\% C.I. 1.000 to 1.000$)$, and IPTW ( $B=0.000$, S.E $=0.000$, Wald $=278.483, \mathrm{P}=0.000, \mathrm{OR}=1.000$, $95 \%$ C.I. 1.000 to 1.000). There were 455 patients 
Table 2. The outcome of using Vitamin D in the treatment of the COVID-19 patients analyzed as all-cohort, propensity score adjustment, and inverted probability of treatment weight (IPTW) by logistic regression analysis.

\begin{tabular}{|c|c|c|c|c|c|c|c|}
\hline \multicolumn{2}{|c|}{ Characteristic } & B & S.E. & Wald & $\mathbf{P}$ & OR (ExpB) & $95 \%$ C.I. \\
\hline \multirow{3}{*}{ Recovery* } & All-cohort & 0.000 & 0.000 & 4.076 & 0.043 & 1.000 & 1 to 1 \\
\hline & IPSW & 0.000 & 0.000 & 187.905 & 0.000 & 1.000 & 1 to 1 \\
\hline & IPTW & 0.000 & 0.000 & 278.483 & 0.000 & 1.000 & 1 to 1 \\
\hline \multirow{3}{*}{$\begin{array}{l}\text { On home } \\
\text { oxygen }\end{array}$} & All-cohort & 0.000 & 0.000 & 16.421 & 0.000 & 1.000 & 1 to 1 \\
\hline & IPSW & 0.000 & 0.000 & 164.703 & 0.000 & 1.000 & 1 to 1 \\
\hline & IPTW & 0.000 & 0.000 & 207.543 & 0.000 & 1.000 & 1 to 1 \\
\hline \multirow{3}{*}{$\begin{array}{l}\text { All-cause } \\
\text { Mortality }\end{array}$} & All-cohort & 0.000 & 0.000 & 4.874 & 0.028 & 1.000 & 1 to 1 \\
\hline & IPSW & 0.000 & 0.000 & 8.386 & 0.004 & 1.000 & 1 to 1 \\
\hline & IPTW & 0.000 & 0.000 & 20.509 & 0.000 & 1.000 & 1 to 1 \\
\hline
\end{tabular}

Percent in (brackets) are proportions ofsub-variables when statistical significance was demonstrated. *2-sided Significance (P-value) was tested by $x 2$, and Bonferroni method.\% Invasive mechanical ventilation. NS: not significant.

discharged home on oxygen, no observable effect of vitamin $D$ to avoid patients form using home oxygen $(B=0.000$, S.E $=0.000$, Wald $=16.421$, $P=0.000, O R=1.000,95 \%$ C.I. 1.000 to 1.000 ), similarly no significance was for IPSM analysis $(B=$ 0.000, S.E $=0.000$, Wald $=164.703, P=0.000$, OR $=1.000,95 \%$ C.I. 1.000 to 1.000$)$, and IPTW (B = 0.000, S.E $=0.000$, Wald $=207.543, P=0.000$, OR $=1.000,95 \%$ C.I. 1.000 to 1.000 ).

Overall, all-cause mortality was $13.79 \%$ (156 patients), and for those who stayed in the ICU the mortality was $55.0 \%$ (110 patients). There was no significant effect for the cumulative doses of vitamin $D$ on all-cause mortality $(B=0.000, S . E=0.000$, Wald $=4.847, P=0.028, O R=1.000,95 \%$ C.I. 1.000 to 1.000$)$, similarly no significance was noted for IPSM analysis $(B=0.000, S . E=0.000$, Wald $=$ 8.386, $P=0.004, O R=1.000,95 \%$ C.I. 1.000 to 1.000), and IPTW (B = 0.000, S.E $=0.000$, Wald $=$ 20.509, $P=0.000, O R=1.000,95 \%$ C.I. 1.000 to 1.000) (Table 2).

\section{Discussion}

With the advent of the COVID-19 pandemic and its high morbidity and mortality including more clot- ting, several organs inflammation, hypoxemia, and acute and chronic interstitial lung diseases that may end in fibrosis in some patients [18-20].Several interventions to alleviate the disease severity are being sought to handle the high morbidity and mortality associated with COVID-19, including several therapeutic interventions, steroids, anticoagulants, antivirals, and vitamin D (25- $(\mathrm{OH})_{2}$ cholecalciferol). Previous studies demonstrated that vitamin $\mathrm{D}$ had a favorable preventive effect in seasonal influenza $A$ infection [21]. However, its therapeutic effects are being disputed until now $[22,23]$.

In our study, we evaluated the prescribed cumulative doses of vitamin $D$, and whether they have effects on alleviating COVID-19 patients, our primary interest was to evaluate the impact of the increasing cumulative doses of vitamin $D$ on the outcomes; recovery by hospital discharge, the need for home oxygen therapy, and all-cause mortality.

Recovery from COVID-19 by hospital discharge as defined here: patients who did not need for home $\mathrm{O} 2$ therapy, and did not have the following symptoms offever, headaches, myalgias, loss of taste, loss of smell, and chills and other symptoms that were non-existent before COVID-19. In all-cohort, IPSW, and IPTW, vitamin D did not improve recovery in 
COVID-19 patients (OR $=1,95 \%$ C.I. 1 to 1$)$. Also, it did not improve the rates of the need for home oxygen therapy for discharged patients $(O R=1$, $95 \%$ C.I. 1 to 1). The all-cause mortality in our patients was $13.79 \%$ and the ICU mortality was 55\%. However, patients analyzed as all-cohorts, IPSW and IPTW did not show any improvement with the increasing vitamin $\mathrm{D}$ cumulative dose $(\mathrm{OR}=1,95 \%$ C.I. 1 to 1$)$.

\section{Conclusion}

In our patients' cohort, we could not demonstrate a beneficial effect for vitamin $D$ as a therapy in COVID-19 patients for the measured outcomes; recovery by hospital discharge, the need for home oxygen therapy, and all-cause mortality. Those results confirm previous literature that demonstrated no clear clinical effects for using vitamin D as a therapy.

\section{References}

1. Alanagreh LA, Alzoughool F, Atoum M. The human coronavirus disease COVID-19: its origin, characteristics, and insights into potential drugs and its mechanisms. Pathogens 2020; (5):331.

2. Nittari G, Pallotta G, Amenta F, Tayebati SK. Current pharmacological treatments for SARS-COV-2: A narrative review. European Journal of Pharmacology 2020; 27:173328.

3. Marik PE, Kory P, Varon J, Iglesias J, Meduri GU. MATH+ protocol for the treatment of SARS-CoV-2 infection: the scientific rationale. Expert Review of Anti-infective Therapy 2021; 19(2):129-35

4. Thomas S, Patel D, Bittel B, Wolski K, Wang Q, Kumar A, II'Giovine ZJ, Mehra R, McWilliams C, Nissen SE, Desai MY. Effect of highdose zinc and ascorbic acid supplementation vs usual care on symptom length and reduction among ambulatory patients with SARS-CoV-2 infection: the COVID A to $Z$ randomized clinical trial. JAMA Network Open 2021; 4(2):e210369-

5. Faqihi F, Alharthy A, Alodat M, Asad D, Aletreby W, Kutsogiannis DJ, Brindley PG, Karakitsos D. A pilot study of therapeutic plasma exchange for serious SARS CoV-2 disease (COVID-19): a structured summary of a randomized controlled trial study protocol. Trials 2020; 21:1-3.
6. Balcells ME, Rojas L, Le Corre N, Martínez-Valdebenito C, Ceballos ME, Ferrés M, Chang M, Vizcaya C, Mondaca S, Huete Á, Castro R. Early versus deferred anti-SARS-CoV-2 convalescent plasma in patients admitted for COVID-19: A randomized phase II clinical trial. PLoS Medicine 2021; 18(3):e1003415.

7. Sundaram ME, Coleman LA. Vitamin D and influenza. Advances in Nutrition 2012; 3(4):517-25.

8. Mitsuyoshi Urashima, TakaakiSegawa, Minoru Okazaki, Mana Kurihara, Yasuyuki Wada, Hiroyuki Ida, Randomized trial of vitamin $D$ supplementation to prevent seasonal influenza $A$ in schoolchildren, The American Journal of Clinical Nutrition 2010; 9: 5, 1255-1260.

9. Science M, Maguire JL, Russell ML, Smieja M, Walter SD, Loeb M. Low serum 25-hydroxyvitamin D level and risk of upper respiratory tract infection in children and adolescents. Clinical Infectious Diseases 2013; 57(3):392-7.

10. Dubnov-Raz G, Hemilä $H$, Cohen AH, Rinat B, Choleva L, Constantini NW. Vitamin D supplementation and upper respiratory tract infections in adolescent swimmers: a randomized controlled trial. Pediatric Exercise Science 2015; 27(1):113-9.

11. Kaufman HW, Niles JK, Kroll MH, Bi C, Holick MF. SARS-CoV-2 positivity rates associated with circulating 25 -hydroxyvitamin $\mathrm{D}$ levels. PloS One 2020; 15(9):e0239252.

12. Castillo ME, Costa LM, Barrios JM, Díaz JF, Miranda JL, Bouillon R, Gomez JM. Effect of calcifediol treatment and best available therapy versus best available therapy on intensive care unit admission and mortality among patients hospitalized for COVID-19: A pilot randomized clinical study. The Journal of Steroid Biochemistry and Molecular Biology 2020; 203:105751.

13. Annweiler C, Hanotte B, de I'Eprevier CG, Sabatier JM, Lafaie L, Célarier T. Vitamin D and survival in COVID-19 patients: a quasi-experimental study. The Journal of Steroid Biochemistry and Molecular Biology 2020; 204:105771.

14. Panfili FM, Roversi M, D'argenio P, Rossi $P$, Cappa M, Fintini D. Possible role of vitamin D in Covid-19 infection in pediatric population. Journal of Endocrinological Investigation 2021; 44(1):27-35.

15. Wong HY, Lam HY, Fong AH, Leung ST, Chin TW, Lo CS, Lui MM, Lee JC, Chiu KW, Chung TW, Lee EY. Frequency and distribution of chest radiographic findings in patients positive for COVID-19. Radiology 2020; 296(2):E72-8.

16. Health Encyclopedia. University of Rochester Medical Center. https://www.urmc.rochester.edu/encyclopedia/content.aspx?c ontenttypeid=167\&contentid=ferritin_blood\#: : :text=The $\% 20$ normal\%20range \%20for \%20ferritin,females \%2C\%2018\%20 to\%2039\%20years. Last accessed 9 October 2021.

17. NCBI resources. Covid-19 information. https://www.ncbi. nlm.nih.gov/books/NBK278991/table/diet-treatment-obes. table4clas/ last accessed 9 October 2021. 
18. Myall KJ, Mukherjee B, Castanheira AM, Lam JL, Benedetti G, Mak SM, Preston R, Thillai M, Dewar A, Molyneaux PL, West AG. Persistent Post-COVID-19 Interstitial Lung Disease. An Observational Study of Corticosteroid Treatment. Annals of the American Thoracic Society 2021; 18(5):799-806.]

19. Pujhari S, Paul S, Ahluwalia J, Rasgon JL. Clotting disorder in severe acute respiratory syndrome coronavirus 2. Reviews in Medical Virology 2021; 31(3):e2177.

20. Fudim M, Qadri YJ, Ghadimi K, MacLeod DB, Molinger J, Piccini JP, Whittle J, Wischmeyer PE, Patel MR, Ulloa L. Implications for neuromodulation therapy to control inflammation and related organ dysfunction in COVID-19. Journal of Cardiovascular Translational Research 2020; 13:894-9.

21. Urashima M, Segawa T, Okazaki M, Kurihara M, Wada $Y$, Ida $H$. Randomized trial of vitamin D supplementation to prevent seasonal influenza A in schoolchildren. The American Journal of Clinical Nutrition 2010; 91(5):1255-60.

22. Teymoori-Rad M, Marashi SM. Vitamin D and Covid-19: From potential therapeutic effects to unanswered questions. Reviews in Medical Virology 2021; 31(2):e2159.

23. Pal R, Banerjee $M$, Bhadada SK, Shetty AJ, Singh B, Vyas A. Vitamin D supplementation and clinical outcomes in COVID-19: a systematic review and meta-analysis. Journal of Endocrino logical Investigation 2021; 24:1-6.

\section{Publish in The International Arabic Journal of Antimicrobial Agents}

The Journal is an open access peer-reviewed journal that publishes scientific papers about all aspects of antimicrobials. The journal will publish original research articles, reviews, brief reports and case reports dealing with basic and clinical antibacterial agents, antiviral, antiprotozoals, antituberculuous, antifungal and antihelminthes agents. All manuscripts must be prepared in English, and are subject to a rigorous and fair peer-review process. Accepted papers will immediately appear online. The journal aims to advance the knowledge, attitude and the research of chemotherapy in the Arabic world in cooperation with international, national scientific and public societies as well as research centers with similar aims and objectives. 